\title{
A Novel Parasitic Satellite Communication System ${ }^{*}$
}

\author{
Junxia Cui, Huli Shi, Chang Lv \\ National Astronomical Observatories of CAS, Beijing, China, 100012 \\ cuijx@nao.cas.cn,.shl@nao.cas.cn, lc@nao.cas.cn
}

\begin{abstract}
In the event of emergencies, if satellites transponders are blocked or destroyed, we can transfer the signal to another transponder or satellite without detection of a third party, which is called satellite parasitic communication. This paper put forward a novel satellite parasitic communication system. This system can meet not only communication hidden nature, but also communication reliability. The characteristic of this system is that it takes use of wideband spread spectrum technology and a novel MSAT (Micro Small Aperture Terminal). Firstly, this novel spread spectrum system parasitic performance is analyzed theoretically. The results show that: (1) the larger number of parasitic spread spectrum signals, the greater decrease of signal-to-noise (SNR); (2) the greater SNR of the business channel, the greater decline of SNR caused by the parasitic; (3) when the business channel SNR and the parasitic number of the spread signal are constant, the higher the interference tolerance of the spread spectrum signal, the smaller the reduction of SNR to the original business signals. At last, taking use of Forwarding-satellite test system resources, this novel parasitic satellite communication system performance test on the television broadcast signal is taken. The results show that the MSAT signal and the broadcast television can work together with good parasitic performance.

Index Terms - satellite navigation; spread spectrum; parasitic communication; anti-jamming; TV broadcast.
\end{abstract}

\section{Introduction}

In a satellite communication system, in order to prevent adjacent satellite interference generated among satellite networks, there are strict regulations by the International Telecommunication Union (ITU) about the earth station offaxis radiation power spectral density, such as ITU-R S.524-8 and power flux density at the surface by satellites, such as ITU-R SF.358-5 $5^{[1,2]}$. In addition, satellite operators will coordinate with each other about adjacent satellite interference and the coordination results are often more demanding than the ITU's ${ }^{[3]}$. Therefore, the C-band communication terminal antenna size is usually not less than 1 meter. Transponder satellite navigation experiment system is integrated satellite navigation and communication system based on geostationary orbit (GEO) and slightly inclined geostationary orbit (SIGSO) satellites ${ }^{[4]}$. There are numerous frequency resources on SIGSO satellites. According to the characteristics of SIGSO satellites, this system has developed a special type of microsatellite communication terminal (MSAT) $)^{[5]}$, of which the antenna is very small, such as whip antenna, monopole beam shaped antenna, and the maximum diameter parabolic antenna of $0.45 \mathrm{~m}$, and transmission power is no more than $10 \mathrm{~W}^{[6]}$. Since the main lobes of these antennas are relatively wide, in order to avoid adjacent satellite interference, it adopts direct sequence spread spectrum signal system. With such low effective isotropic radiated power (EIRP) and low power spectral density, there is some probability for the MSAT spread spectrum signal to be parasitic on the other communication signals.

MSAT spread spectrum signal parasitic performance is analyzed theoretically at first. And then, performance verification of MSAT communication signal parasitic on satellite TV signal is carried out, utilizing Apstar-1 satellite and $16 \mathrm{~m}$-diameter antenna ground station. Results show that MSAT spreading spectrum signal has a good parasitic performance even if it shares the same center frequency with an ordinary satellite TV signal, while keeping satellite TV broadcasting un-disturbed and error free transmission with the error rate below $10^{-6}$.

\section{MSAT Spread Spectrum Signal Parasitic Performance Theoretical Analysis}

Satellite parasitic communication is necessary to meet communication concealed nature, but also to meet the communication reliability. The concealed nature of satellite parasitic communication refers that not only the parasitic signal will not form an obvious interference for the original business signal, but it also does not change the main communication characteristics concerned by satellite monitoring system and the business signal users. In accordance with the characteristics of the satellite communication, when it is affected by weather, environmental changes, satellite link instability or other factors, background noise and SNR within the transponder frequency band tends to fluctuate within a certain range. Tests show that when the weather conditions are good, the background noise and SNR change is about $1 \mathrm{~dB}^{[7]}$. However, under worse weather conditions, background noise and SNR get worse. Satellite parasitic communication takes use of such feature to keep the variation by parasitic signal not exceeding the natural range of background noise and SNR. In this way, the parasitic communication purpose can be achieved. Thus, the business signal SNR decrease caused by the parasitic communication becomes an important indicator of concealment ability measurement of the MSAT spread spectrum parasitic communications signal.

We assume satellite original business channel SNR as Q under the condition of the normal communication (stable channel and no other signal interference), and interference tolerance of the spread spectrum signal for parasitic communication as $\mathrm{M}_{\mathrm{wj}}$. Take channel $\mathrm{SNR}$ of $\mathrm{Q}$ as the

\footnotetext{
* This work is supported by NSFC Grant \#61001109 to Junxia Cui.
} 
indicator to measure channel performance. Interference tolerance of the spread spectrum signal is stated as

$$
\mathrm{M}_{w j}=10 \lg \frac{J}{P}=10 \lg \frac{W}{R}-10 \lg \frac{E_{b}}{J_{0}}
$$

where $\mathrm{J}$ represents the maximum interference power; $\mathrm{P}$ represents MSAT spread spectrum signal power; $\mathrm{W}$ represents spread spectrum bandwidth; $\mathrm{R}$ is the data rate; $\mathrm{W} / \mathrm{R}$ indicates the spread spectrum processing gain; $\mathrm{E}_{\mathrm{b}} / \mathrm{J}_{0}$ is representative of SNR threshold. For MSAT satellite communication system, R of is $700 \mathrm{bps} ; \mathrm{W}$ is $4.2966 \mathrm{Mcps} ; \mathrm{W} / \mathrm{R}$ is $37.9 \mathrm{~dB}$, and $\mathrm{E}_{\mathrm{b}} / \mathrm{J}_{0}$ is 6.5dB. Considering system loss of $2.5 \mathrm{~dB}$, we can get that $\mathrm{M}_{\mathrm{wj}}$ is $28.9 \mathrm{~dB}$. Spread spectrum signal carrier-to-noise ratio $\mathrm{Q}_{1}=$ $10 \lg (\mathrm{P} / \mathrm{N})=-28.31 \mathrm{~dB}$.

In normal communication, the original satellite channel SNR is stated as follow:

$$
Q=10 \lg \left(\frac{P_{s}}{N}\right),
$$

where $\mathrm{N}$ represents channel noise power, Ps represents the original business signal SNR. Then, we can get

$$
N=\frac{P_{s}}{10^{0.1 Q}} .
$$

In this case, the satellite channel original business SNR is

$$
S N R_{0}=10 \lg \frac{P_{s}}{\left(\frac{N}{B_{n}}\right) B_{s}}=Q-10 \lg r
$$

where $r$ indicates the ratio of original business channel bandwidth and satellite transponder bandwidth, that is to say $\mathrm{r}=\mathrm{B}_{\mathrm{s}} / \mathrm{B}_{\mathrm{n}}$.

Influence on business signal SNR is analyzed below when $\mathrm{k}$ of MSAT spread spectrum signals are parasitic on business channel.

In order to receive the spread spectrum signal normally, there should be

$$
10 \lg \left[\frac{\left(\mathrm{P}_{s}+N\right)}{P_{W D S}}\right] \leq M_{W j},
$$

where PWDS represents single spread spectrum signal power. Because Ps is far less than N, the above formula can be changed into formula (4):

$$
10 \lg \left[\frac{N}{P_{W D S}}\right]=M_{W j} .
$$

Assuming that every spread spectrum signal power is equal and the business channel is white Gaussian noise additive, when $\mathrm{k}$ of MSAT spread spectrum signals are superimposed in the business channel at the same time, the actual business signal SNR is stated as:

$$
\mathrm{SNR}_{\mathrm{w}}=-10 \lg r\left(\frac{1}{10^{0.1 Q}}+\frac{k}{10^{0.1 w_{w j}}}\right),
$$

and business signal SNR decreases by

$$
\Delta S N R_{W}=Q+10 \lg \left(\frac{1}{10^{0.1 Q}}+\frac{k}{10^{0.1 W_{w j}}}\right) .
$$

According to formula (6), Fig. 1(1) - (6) are given to show the relationship curves of actual business signal SNR in parasitic communication and the original channel SNR, given that $\mathrm{M}_{\mathrm{wj}}$ is $10 \mathrm{~dB}, 20 \mathrm{~dB}, 28.95 \mathrm{~dB}, 40 \mathrm{~dB}, 50 \mathrm{~dB}, 60 \mathrm{~dB}$ and spread spectrum signal quantity $\mathrm{k}$ is $1,5,10,20$, or 30 respectively.
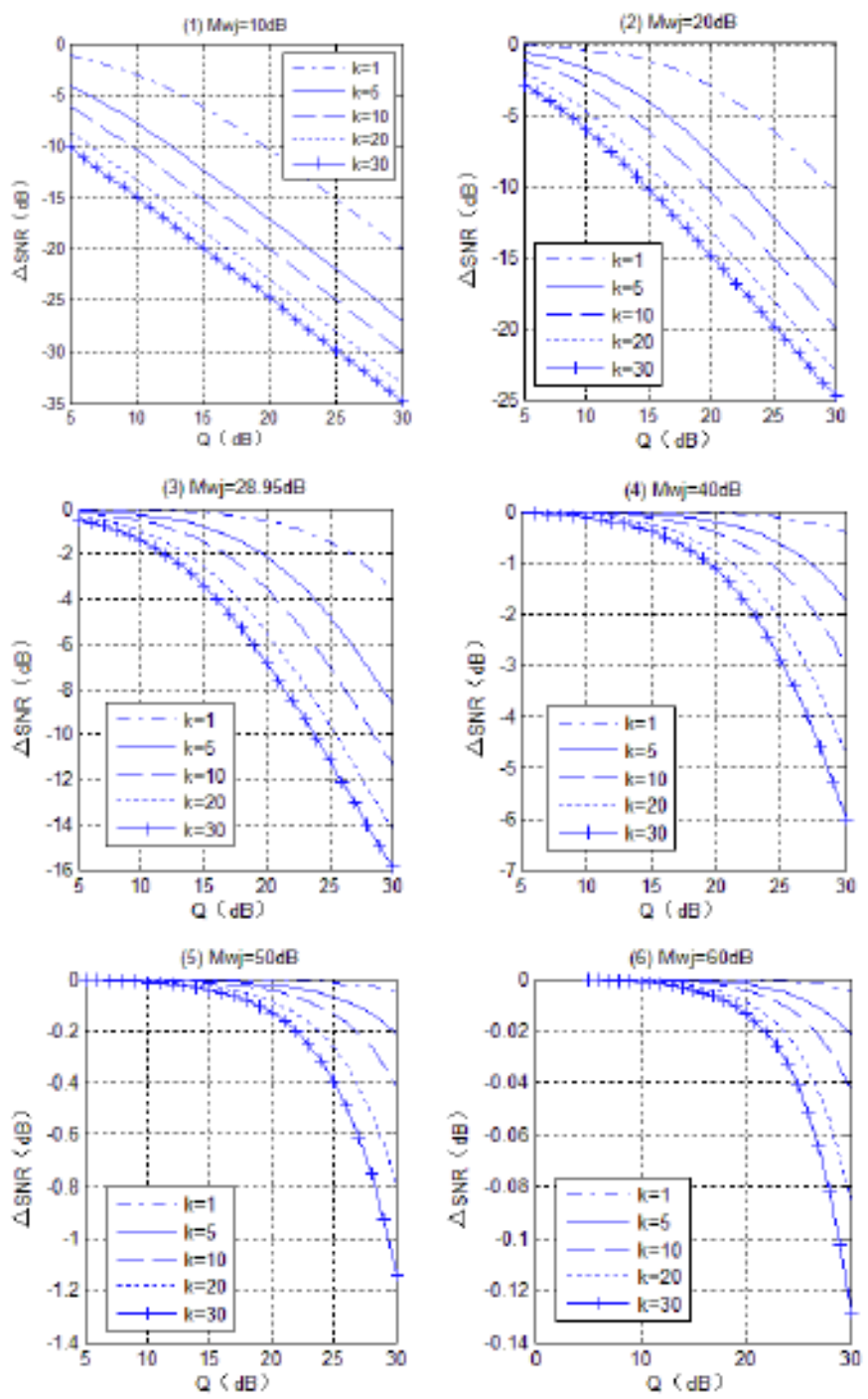

Fig. 1 Relationship between SNR change of business signal in parasitic communication and the original business channel SNR

Three conclusions can be drawn from Fig. 1. Firstly, the larger parasitic spread spectrum signals quantity of $\mathrm{k}$, the greater decrease of business signal SNR. Secondly, the stronger the original business channel SNR of Q, the more decline of business signal SNR caused by the parasitic. Thirdly, when the original business channel SNR of Q and the parasitic number of the MSAT spread spectrum signals are constant, the higher the interference tolerance of the spread 
spectrum signal of $\mathrm{M}_{\mathrm{wj}}$, the smaller reduction of business signal SNR.

Therefore, if the following three conditions are met, it is possible to achieve the best parasitic communication performance: (1) the original business channel SNR of $Q$ is lower; (2) parasitic spread spectrum signal interference tolerance of $\mathrm{M}_{\mathrm{wj}}$ is as large as possible; (3) the number of parasitic spread spectrum signal is small.

\section{Test and Verification of MSAT Spread Spectrum Signal Parasitic on Satellite TV Signal}

In order to verify MSAT spread spectrum signal parasitic performance on the satellite television signal, we take use of Apstar-1 satellite located at $142^{\circ} \mathrm{E}$ for the experiment. We transmit MSAT spread spectrum communication signal at central frequency of $6145 \mathrm{MHz}$ which is the same as of satellite TV signal, to examine whether MSAT spread spectrum signal has impact on reception of satellite TV signal (parasitic performance) and whether satellite TV signal has impact on MSAT signal (anti-interference performance). Parasitic spread spectrum signal is transmitted by $0.45 \mathrm{~m}$-diameter aperture MSAT and received by the $16 \mathrm{~m}$-diameter aperture antenna communication center station. Satellite TV signal is transmitted and received by $16 \mathrm{~m}$-diameter aperture antenna through Apstar-1 satellite. The experiment field is shown in Fig. 2. And the experiment diagram is shown in Fig.3.

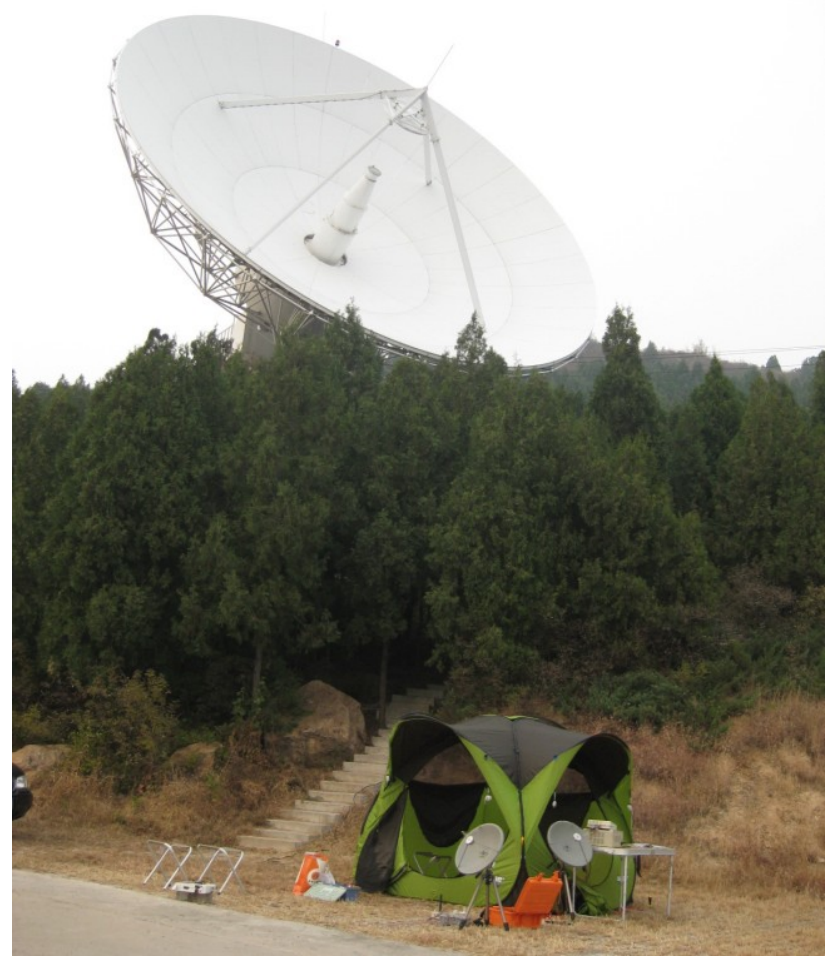

Fig.2 Experiment field

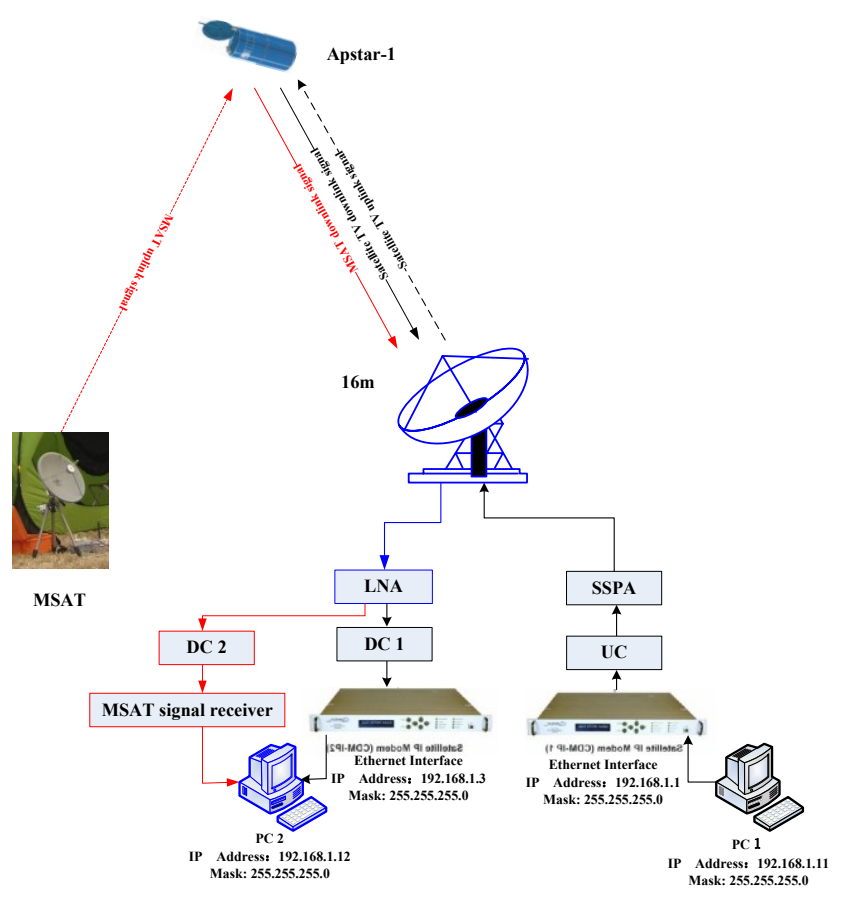

Fig. 3 MSAT communication signal parasites on satellite television signal experiment diagram

Satellite TV signal parameters are shown in Table 1. Parasitic MSAT spread spectrum signal parameters are shown in Table 2.

TABLE 1 Experiment Satellite TV Signal Parameters

\begin{tabular}{|c|c|c|c|}
\hline Item & Value & Item & Value \\
\hline Uplink frequency & $6145 \mathrm{MHz}$ & $\begin{array}{c}\text { Downlink } \\
\text { frequency }\end{array}$ & $3920 \mathrm{MHz}$ \\
\hline Modulation & QPSK & Data rate & $2.048 \mathrm{Mbps}$ \\
\hline Video coding & H.264 & $\begin{array}{c}\text { Transmit antenna } \\
\text { gain }\end{array}$ & $58.0 \mathrm{dBi}$ \\
\hline Audio coding & MPEG 4 Audio & $\begin{array}{c}\text { Receive antenna } \\
\text { gain }\end{array}$ & $54.1 \mathrm{dBi}$ \\
\hline FEC & $3 / 4$ Viterbi & EIRPe & $43.7 \mathrm{dBw}$ \\
\hline Transmit power & $-14.3 \mathrm{dBm}$ & $\begin{array}{c}\text { Demodulation } \\
\text { threshold } \mathrm{E}_{\mathrm{b}} / \mathrm{N}_{0 \mathrm{th}}\end{array}$ & $2 \mathrm{~dB}$ \\
\hline
\end{tabular}

TABLE 2 MSAT Spread Spectrum Signal Parameters

\begin{tabular}{|c|c|c|c|}
\hline Item & Value & Item & Value \\
\hline Uplink frequency & $6145 \mathrm{MHz}$ & Data rate & $700 \mathrm{bps}$ \\
\hline $\begin{array}{c}\text { Downlink } \\
\text { frequency }\end{array}$ & $3920 \mathrm{MHz}$ & Modulation & QPSK \\
\hline $\begin{array}{c}\text { Spreading code } \\
\text { rate }\end{array}$ & $4.2966 \mathrm{Mcps}$ & Bandwidth & $6 \mathrm{MHz}$ \\
\hline $\begin{array}{c}\text { Spreading code } \\
\text { length }\end{array}$ & 1023 & $\begin{array}{c}\text { MSAT transmit } \\
\text { power }\end{array}$ & $1 \mathrm{~W}$ \\
\hline $\begin{array}{c}\text { Transmit antenna } \\
\text { gain(0.45m) }\end{array}$ & $25 \mathrm{dBi}$ & EIRPe & $25 \mathrm{dBw}$ \\
\hline $\begin{array}{c}\text { Receive antenna } \\
\text { gain }(16 \mathrm{~m})\end{array}$ & $54.1 \mathrm{dBi}$ & $\begin{array}{c}\text { Demodulation } \\
\text { threshold } \mathrm{E}_{\mathrm{b}} / \mathrm{N}_{\text {th }}\end{array}$ & $9.5 \mathrm{~dB}$ \\
\hline
\end{tabular}


Interference tolerance $\mathrm{M}_{\mathrm{wj}}$ of spreading spectrum signal is $28.95 \mathrm{~dB}$; parasitic spreading spectrum number $\mathrm{k}$ is 1 ; and satellite TV signal original channel SNR of Q is 9dB. Fig. 1 (3) shows that satellite TV SNR decreases caused by parasitic MSAT signal are very small. If the above parameters are substituted into the formula (6), we can get $\triangle \mathrm{SNR}$ of $0.0449 \mathrm{~dB}$. Actual test results are shown in Fig. 4 and Fig. 5. In Fig. 3, the bottom is the received satellite television video; the upper are the MSAT data received. In Fig. 4, the single-tone carrier is from MSAT; while the broader is TV carrier received.

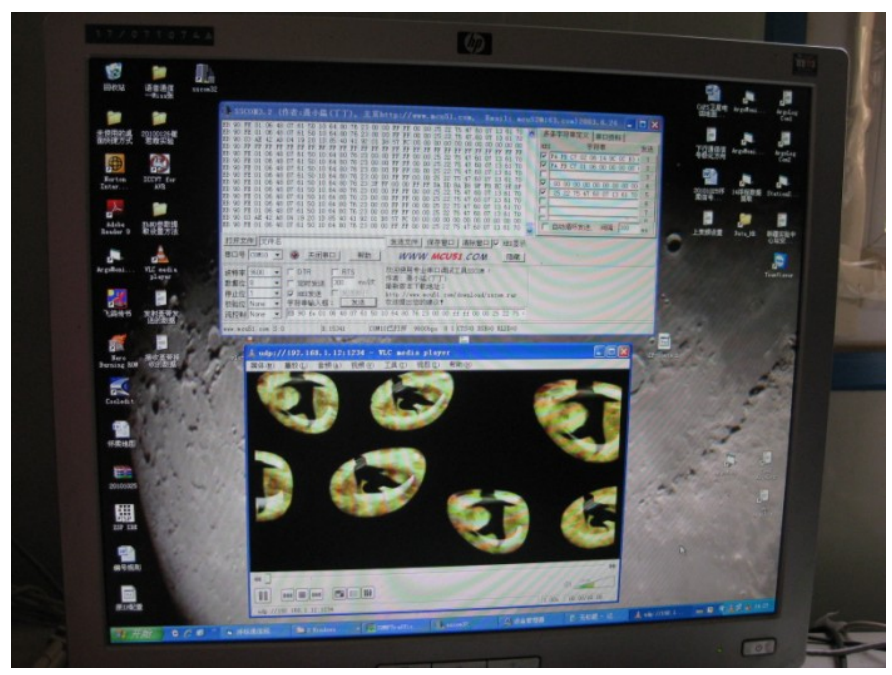

Fig. 4 Satellite TV video and MSAT data received

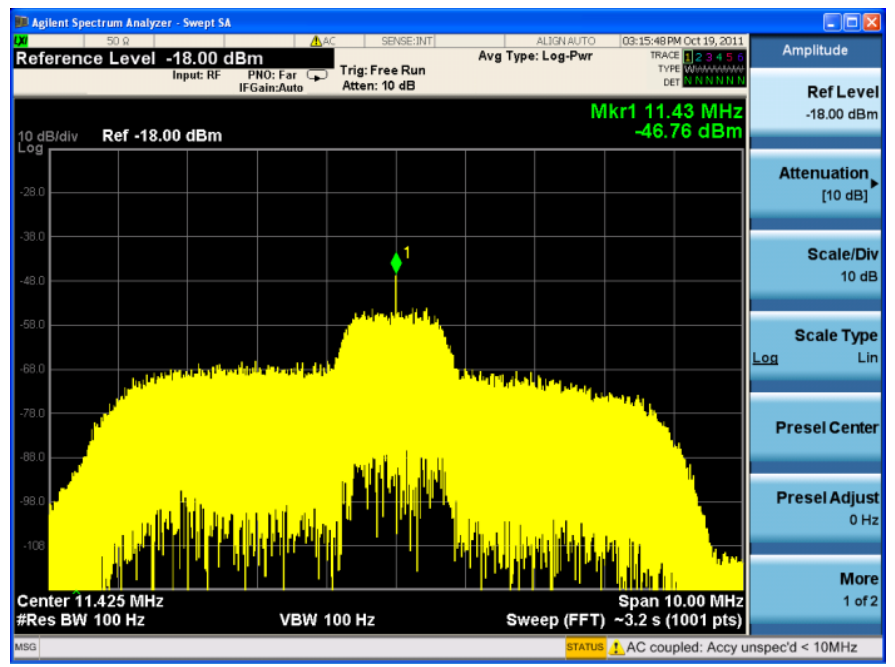

Fig. 5 Satellite TV and MSAT single-tone carrier received
By the formula (6), it can also be calculated that if $\triangle \mathrm{SNR}$ is $1 \mathrm{~dB}$, there are 25 spread spectrum signals can be parasitic on this satellite TV signal. That is to say, there are up to 25 MSAT spread spectrum signals can be parasitic on this satellite TV signal without detection.

\section{Conclusions}

A novel parasitic satellite communication system is put forward in this paper. Firstly, its parasitic performance is analyzed theoretically in detail, and we get a conclusion that if we choose a lower SNR business channel and a few parasitic spread signals with interference tolerance as large as possible, we can get a good parasitic communication performance. At last, performance verification of MSAT communication signal parasitic on satellite TV signal is carried out, utilizing Apstar1 satellite and 16m-diameter antenna center station. Results show that MSAT spreading spectrum signal has a good parasitic performance even if it shares the same center frequency with an ordinary satellite TV signal, while keeping satellite TV broadcasting undisturbed and error free transmission with the error rate below 10-6, and there are up to 25 MSAT spread spectrum signals can be parasitic on the satellite TV signal.

\section{References}

[1] International Telecommunication Union. Recommendation ITU-R S.524-9. Maximum permissible levels of off-axis e.i.r.p density from earth stations in geostationary-satellite orbit networks operating in the fixed-satellite service transmitting in the $6 \mathrm{GHz}, 13 \mathrm{GHz}, 14 \mathrm{GHz}$ and $30 \mathrm{GHz}$ frequency bands. Geneva: International Telecommunication Union Electronic Publishing Service, 2006

[2] International Telecommunication Union. Recommendation ITU-R SF.358-5. Maximum power flux-density at the surface of the Earth by emissions produced by satellites in the fixed satellite service using the same frequency bands above $1 \mathrm{GHz}$ as line-of-sight radio relay systems. Geneva: International Telecommunication Union Electronic Publishing Service, 1995

[3] Cui Junxia, Shi Huli, Chen Jibin, Regulations of spectrum density in adjacent satellites. Satellite \& network, 2008, (1/2):66-67.

[4] Ai Guoxiang, Shi Huli, Wu Haitao, et al. The principal of the positioning system based on communication satellites. Sci China Ser GPhys Mech Astron, 2009, 52(3):472-488

[5] Cui Junxia, Shi Huli and Pang Feng. Azimuth and Elevation of Slightly Inclined Geostation-ary-satellite Orbits , 2009 International Conference on Computer Engineering and Technology (ICCET 2009). Singapore, 2009: 350-352

[6] Cui JunXia, Shi HuLi, Chen Jibin \& PEI Jun. The transmission link of CAPS navigation and communication system. Science in China Series G: Physics, Mechanics \& Astronomy, 2009, 52(3) : 402-411

[7] Li Hongtao, Hao Shiqi, Wang Yingchun, Choosing Data Rate and Channel of the Concealed Satellite Communication, J. of Modern Military Communication, 2005, 13(1), P8-10. 